\title{
CONCEPTS AND EMERGING TRENDS IN MANAGEMENT OF LOCALLY ADVANCED BREAST CANCER
}

\author{
Puneet Kumar Agarwal ${ }^{1}$, Richa Goyal ${ }^{2}$
}

${ }^{1}$ Associate Professor, Department of Surgery, AIIMS, Bhopal, Madhya Pradesh, India.

2Medical Officer, Department of Radiodiagnosis, AIIMS, Bhopal, Madhya Pradesh, India.

\section{BACKGROUND}

ABSTRACT

LABC (Locally Advanced Breast Cancer) comprises of a subset of invasive breast malignancies, where the clinical and radiological assessment ascertains confinement of the disease to breast and regional lymph nodes. The present article, besides discussing the diagnostic workup and assessment of LABC, also covers in detail the different treatment modalities and their controversial role in treatment of LABC. Also discussed are different surgical options and options for breast reconstruction following mastectomy.

\section{KEY WORDS}

Management, Locally Advanced Breast Cancer.

HOW TO CITE THIS ARTICLE: Agarwal PK, Goyal R. Concepts and emerging trends in management of locally advanced breast cancer. J. Evolution Med. Dent. Sci. 2018;7(26):3071-3076, DOI: 10.14260/jemds/2018/689

\section{BACKGROUND}

Patients with locally advanced breast cancer include those with large primary tumours $(>5 \mathrm{~cm})$. Tumours involving the chest wall, skin involvement, ulceration or satellite skin nodules, inflammatory carcinoma, bulky or fixed axillary nodes and clinically apparent internal mammary or supraclavicular nodal involvement.

LABC comprises of a subset of invasive breast malignancies where the clinical and radiological assessment ascertains confinement of the disease to breast and regional lymph nodes.

As per recommendation of NCC Guidelines 2014 that LABC is represented by stage III category of AJCC clinical staging. Patient with stage III disease may be further divided into-

\section{Operable LABC (T3N1M0)}

Those where initial surgical approach is likely to achieve pathologically negative margins and provide long-term control.

\section{Inoperable LABC}

(Clinical stage IIIA [Except for T3N1M0], clinical stage IIIB or clinical stage IIIC), those where initial surgical approach is unlikely to successfully remove all disease and provide longterm control.

\section{Diagnosis and Assessment(1,2,3)}

- The workup includes history and physical examination followed by investigations like CBC, LFT with alkaline phosphatase tests, chest imaging, pathology review and pre-chemotherapy determination of tumour ER/PR receptor status and HER-2 status.

- Imaging modalities routinely include B/L Mammograms, Ultrasonomammogram of breast.

- Breast MRI is recommended for mammographically occult tumours in suspicious cases only.

'Financial or Other Competing Interest': None.

Submission 11-05-2018, Peer Review 10-06-2018,

Acceptance 16-06-2018, Published 25-06-2018.

Corresponding Author:

Dr. Puneet Kumar Agarwal,

House No. 5022, AIIMS, Bhopal Campus, Saket Nagar,

AIIMS, Bhopal-462020, Madhya Pradesh, India.

E-mail: drpuneetk2012@gmail.com

DOI: $10.14260 /$ jemds $/ 2018 / 689$

\section{(c) (i) $\$$}

- For systemic staging bone scan abdominal imaging as USG abdomen, CT chest with abdomen and MRI abdomen are to be tailored as per patient's signs and symptoms.

- FDG PET scan is indicated when standard imaging modalities are inconclusive.

- Fertility counselling should be done in premenopausal women.

- Genetic counselling is recommended for high-risk hereditary breast cancer.

\section{Prognostic and Predictive Factors $(4,5,6,7)$ Primary Tumour Size}

Studies demonstrate that with increasing tumour size, recurrence of breast cancer at distant sites is more common and Overall Survival (OS) is reduced.

\section{Lymph Node (LN) Status}

In women with positive LNs, there is a direct correlation between the number of LNs affected and the risk of disease recurrence.

\section{Pathologic Tumour Characteristics}

Mitotic index, differentiation and pleomorphism of cancer cells grade the tumour and assess its aggressiveness.

\section{Hormonal Receptor Status}

The presence of HRs on invasive breast cancer cells is both prognostic and predictive. HR-positive breast cancer responds well with endocrine therapy as compared to HRnegative disease. Dividing HR status into oestrogen receptor (ER)-positive and progesterone positive further estimates response to endocrine therapy.

\section{HER-2 Status}

Proto-oncogene HER-2 overexpression on breast cancer cells (20\% to $25 \%$ of cases) is associated with tumour aggressiveness, recurrence rates and mortality, especially in women with LN-positive disease.

Tumours with HER-2 overexpression are relatively resistant to endocrine therapy alone. These women appear to respond better to an aromatase inhibitor (AI) compared with tamoxifen; are relatively resistant to certain chemotherapy regimens (e.g. cyclophosphamide, methotrexate and 
fluorouracil [CMF]); and respond better to anthracycline and taxane-based chemotherapy regimens. These tumours also respond well to trastuzumab therapy, which is targeted against the extracellular domain of this human epidermal growth factor receptor.

\section{Proliferation Markers( 8,9$)$}

Women with high concentrations of cyclin E, in particular, have reduced 5-year DFS and OS rates. Increased expression of another proliferation marker, Ki-67, may also correlate with decreased Overall Survival (OS) and Disease Free Survival (DFS).

Use of DNA microarray(10) technologies to characterise breast cancer has allowed for development of classification systems of breast cancer by gene expression profile. Five major subtypes of breast cancer have been identified by DNA microarray gene expression profiling: ER-positive/HER2negative (luminal $\mathrm{A}$ and luminal $\mathrm{B}$ subtypes); ERnegative/HER2-negative (basal subtype); HER2-positive and tumours that have characteristics similar to normal breast tissue. In retrospective analyses, these gene expression subtypes are associated with differing relapse-free survival and OS.

\section{CD3+ Tumour Infiltrating Lymphocytes}

Recent studies have shown that detection of density and location of CD3+ tumour infiltrating lymphocytes could be an independent prognostic marker. But further studies with large sample size are needed to corroborate this.(11)

\section{Treatment of Non-Inflammatory Locally Advanced Breast Cancer

Operable LABC (T3N1M0)(12,13,14,15)

For patients desirous for BCS, core biopsy of lump with placement of image detectable markers for demarcating tumour bed is done.

For axillary disease, FNAC or core biopsy with placement of image detectable marker is done for clinically positive lymph nodes and for clinically negative nodes axillary ultrasound is done and suspicious nodes should be sampled by FNA or core biopsy and clipped with image-detectable marker.

After administration of preoperative systemic therapy, assessment is done.

- If no response or partial response, proceed to mastectomy.

- Complete response or partial response amounting to $\mathrm{BCS}$, proceed to lumpectomy.

Those patients who fail to respond to preoperative systemic therapy will undergo mastectomy followed by reconstruction.

For axillary disease, candidates with clinically positive nodes with positive result of nodal FNAC or core biopsy will undergo axillary LN I/II dissection.

Patients with clinically negative nodes and those who have clinically positive nodes, but with nodal FNAC or core biopsy negative will undergo sentinel lymph node biopsy.

Candidates with sentinel positive LN or sentinel LN not identified should be considered for axillary LN I/II dissection. If sentinel lymph node is negative, no further surgery is required. Level III dissection to the thoracic inlet should be performed only in cases with gross disease in level II.

In the absence of gross disease in level II nodes, lymph node dissection should include tissue inferior to the axillary vein from the latissimus dorsi muscle laterally to the medial border of the pectoralis minor muscle (Level I/II).

Adjuvant chemotherapy and endocrine therapy (for ER/PR positive) with radiation therapy to whole breast with or without boost to tumour bed, supra and infraclavicular lymph nodes and with strong consideration for internal mammary nodes follows.

Candidates not desirous for BCS will undergo total mastectomy with surgical axillary staging followed by adjuvant chemotherapy and radiation therapy to chest wall, supra and infraclavicular lymph nodes and also internal mammary nodes.

Delayed breast reconstruction follows.

\section{Inoperable LABC(16)}

(Clinical stage IIIA [Except for T3N1M0], clinical stage IIIB or Clinical stage IIIC)

Patients presenting with inoperable non-inflammatory, locally advanced disease at presentation, the initial use of anthracyclines based preoperative systemic therapy with or without a taxane is standard therapy.

Patients with LABC that is HER2-positive should receive an initial chemotherapy programme that incorporates trastuzumab.

Local Therapy following a Clinical Response to Preoperative Systemic Therapy consists of-

1. Total mastectomy with level I/II ALN dissection with or without delayed breast reconstruction, or

2. Lumpectomy and level I/II axillary dissection.

Both local treatment groups are considered to have sufficient risk of local recurrence, which needs the use of chest wall (breast) and supraclavicular nodes irradiation. If internal mammary nodes are involved, they should also be irradiated. Without detected internal mammary node involvement, consideration may be given to include the internal mammary nodes in the radiation field. Adjuvant therapy may involve completion of planned chemotherapy regimen if not completed preoperatively followed by endocrine therapy in patients with HR status positive.

Patients with inoperable stage III tumour with disease progression during preoperative systemic therapy should be considered for palliative breast irradiation in an attempt to enhance local control.

In all subsets of patients, further systemic adjuvant chemotherapy after local therapy is felt to be standard. Tamoxifen (or an Aromatase inhibitor if menopausal) should be added for those with hormone receptor positive tumours and trastuzumab to HER2-positive tumours.

\section{Surveillance and Follow-Up(17,18,19)}

This Comprises of the following:

- History and physical exam every 4 - 6 months for $5 \mathrm{y}$, then every 12 months.

- Mammography every 12 months.

- Women on tamoxifen: annual gynaecologic assessment every 12 months if uterus present. 
- Women on an aromatase inhibitor or who experience ovarian failure secondary to treatment should have monitoring of bone health with a bone mineral density determination at baseline and periodically thereafter.

- Assess and encourage adherence to adjuvant endocrine therapy.

- $\quad$ Evidence suggests that active lifestyle and achieving and maintaining an ideal body weight (20 - $25 \mathrm{BMI}$ ) may lead to optimal breast cancer outcomes.

\section{Inflammatory Breast Carcinoma as LABC}

Inflammatory breast cancer is a clinical syndrome in women with invasive breast cancer, which is characterised by erythema and dermal oedema (peau d'orange) of a third or more of the skin of the breast and with a palpable border to the erythema. The differential diagnosis includes cellulitis of the breast or mastitis. Pathologically, a tumour is typically present in the dermal lymphatics of the involved skin, but dermal lymphatic involvement is neither required nor sufficient by itself for a diagnosis of inflammatory breast cancer.

\section{Diagnosis and Workup for IBC(20)}

The diagnostic workup for inflammatory LABC is similar to non-inflammatory LABC, which includes history and physical examination, CBC, LFT, determination of HR status and HER-2 status breast MRI is indicated in multicentric disease. Ultrasonography, Bone scan, CT scan of chest and abdomen is recommended and FDG-PET scan in suspicious cases.

\section{Treatment (IBC) ${ }^{(21,22)}$}

Pre-operative chemotherapy is started as Anthracycline with Taxane. If the tumour is found to be HER-2 positive, Trastuzumab containing regimen is initiated.

The response to pre-operative chemotherapy determines the type of dissection and following management.

If the response is favourable, then proceed with total mastectomy with level I and II axillary dissection with radiation therapy to chest wall and supraclavicular nodes and internal mammary nodes, if later are positive too. Immediate reconstruction is contraindicated in the setting of mastectomy for inflammatory breast cancer (IBC) due to the high risk of recurrence, aggressive nature of the disease and consequent need to proceed expeditiously to postoperative radiotherapy for local control without any potential delay. As skin-sparing mastectomy has not yet been demonstrated to be safe for IBC, there is also a need to resect currently or previously involved skin at the time of mastectomy and thus no advantage to immediate reconstruction in this setting.

Post-operatively, the chemotherapy regimen is completed along with endocrine treatment if HR positive tumour is encountered. If the tumour is also HER-2 positive, then 1 year of trastuzumab therapy is administered which may be done concurrently with radiation or endocrine therapy.

\section{Treatment Modalities}

\section{Neoadjuvant Chemotherapy(23,24,25)}

\section{Aim is to-}

- Downstage the tumour and involved nodes to achieve operability or failing this to reduce tumour volume for radiotherapy.

- Use the tumour as an in vivo measure of chemosensitivity.

- Achieve early treatment of micrometastases.

\section{Neoadjuvant Endocrine Therapy}

Neoadjuvant Endocrine Therapy is promising for ER positive locally advanced breast cancer. Studies suggest that Aromatase inhibitors such as Letrozole are more effective than tamoxifen.

\section{Surgical Treatment}

It comprises of breast conserving surgery and mastectomy.

\section{Breast Conservation Surgery (BCS)}

Exclusion criteria for breast-conserving therapy requiring radiation therapy include:

\section{Absolute}

- Radiation therapy during pregnancy.

- Diffuse suspicious or malignant-appearing microcalcifications.

- Widespread disease that cannot be incorporated by local excision through a single incision that achieves negative margins with a satisfactory cosmetic result.

- Positive pathologic margin.

- Inflammatory (T4d) disease before neoadjuvant therapy and incomplete resolution of skin involvement after neoadjuvant therapy.

\section{Relative}

- Prior radiation therapy to the chest wall or breast; knowledge of doses and volumes prescribed is essential.

- Active connective tissue disease involving the skin (Especially Scleroderma and Lupus).

- Tumours $>5 \mathrm{~cm}$

- Women with a known or suspected genetic predisposition to breast cancer may have an increased risk of ipsilateral breast recurrence or contralateral breast cancer with breast-conserving therapy.

- Prophylactic bilateral mastectomy for risk reduction may be considered.

- Focally positive margin.

The use of breast-conserving therapy is predicted on achieving a pathologically negative margin of resection. A positive margin should generally undergo further surgery, either a re-excision to achieve a negative margin or a mastectomy.

If re-excision is technically feasible to allow for breastconserving therapy, this can be done with resection of the involved margin guided by the orientation of the initial resection specimen or re-excision of the entire original excision cavity.

It may be reasonable to treat selected cases with breastconserving therapy with a microscopically focally positive margin in the absence of an extensive intraductal component.

For these patients, the use of a higher radiation boost dose to the tumour bed should be considered.

Margins should be evaluated on all surgical specimens from breast-conserving surgery.

\section{Requirements for Optimal Margin Evaluation include:}

- Orientation of the surgical specimens.

- Description of the gross and microscopic margin status.

- Reporting of the distance, orientation and type of tumour (invasive or DCIS) in relation to the closest margin. 


\section{Mastectomy(26)}

\section{Indications}

Certain tumours still require mastectomy including those that are large relative to breast size, those with extensive calcifications on mammography, tumours for which clear margins cannot be obtained on wide local excision and patients with contraindications to breast irradiation. Contraindications to the use of radiation therapy include previous breast or chest wall irradiation, active lupus or scleroderma and pregnancy, although many patients pregnant at diagnosis can complete their pregnancy and receive radiation therapy after delivery.

Patient's preference for mastectomy or a desire to avoid radiation is also a valid indication for mastectomy.

Different types of mastectomy procedures have developed over time, which includes total mastectomy; skinsparing mastectomy and most recently nipplesparing mastectomy. When used with other regional strategies, mastectomy maintains fair OS and Disease Free Survival (DFS).

\section{Adjuvant Endocrine Therapy}

Adjuvant treatment of breast cancer by hormonal manipulation, such as ovarian ablation and suppression and more recently selective ER modification or aromatase inhibition improves $\mathrm{OS}$, decreases annual recurrence rates.

Premenopausal women are offered treatment with tamoxifen with or without ovarian suppression (with goserelin or leuprolide) or ablation.

Postmenopausal women have several treatment options. Tamoxifen with an AI or sequential use of both for a total of 5 years of treatment is optimal.

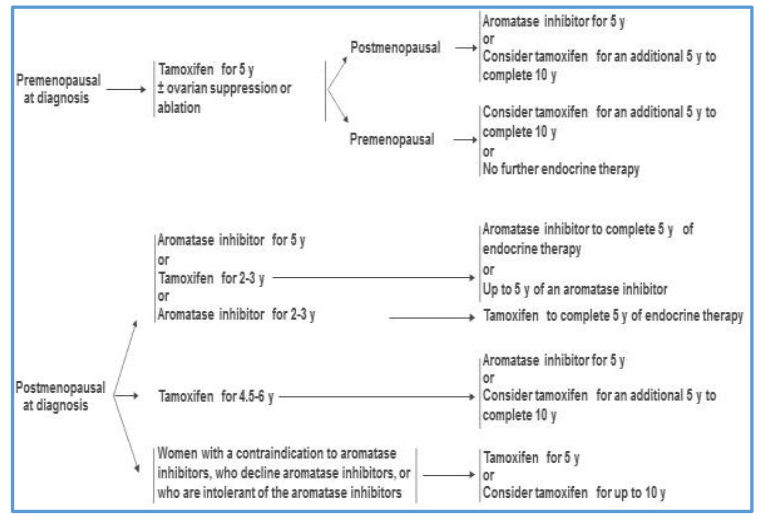

Adjuvant Chemotherapy Regimens(24,25,26)

Reqimens for HER2-neqative disease
Preferred reqimens:
- Dose-dense AC (doxorubicin/cyclophosphamide) followed by
paclitaxel every 2 weeks
- Dose-dense AC (doxorubicin/cyclophosphamide) followed by
weekly paclitaxel
- TC (docetaxel and cyclophosphamide)
Other reqimens:
- Dose-dense AC (doxorubicin/cyclophosphamide)
- FAC/CAF (fluorouracil/doxorubicin/cyclophosphamide)
- FEC/CEF (cyclophosphamide/epirubicin/fluorouracil)
- CMF (cyclophosphamide/methotrexate/fluorouracil)
- AC followed by docetaxel every 3 weeks
- AC followed by weekly paclitaxel
- EC (epirubicin/cyclophosphamide)
- FEC/CEF followed by T
(fluorouracil/epirubicin/cyclophosphamide followed by docetaxel) or
(fluorouracil/epirubicin/cyclophosphamide followed by weekly paclitaxel)
- FAC followed by T
(fluorouracil/doxorubicin/cyclophosphamide followed by weekly
paclitaxel)
- TAC (docetaxel/doxorubicin/cyclophosphamide)

Regimens for HER2-positive disease

Preferred reqimens:

- AC followed by $T+$ trastuzumab \pm pertuzumab

(doxorubicin/cyclophosphamide followed by paclitaxel plus

trastuzumab \pm pertuzumab, various schedules)

- TCH (docetaxel/carboplatin/trastuzumab) \pm pertuzumab

Other reqimens:

- AC followed by docetaxel + trastuzumab \pm pertuzumab

- FEC followed by docetaxel + trastuzumab + pertuzumab

- FEC followed by paclitaxel + trastuzumab + pertuzumab

- Paclitaxel + trastuzumab

- Pertuzumab + trastuzumab * docetaxel followed by FEC

- Pertuzumab + trastuzumab + paclitaxel followed by FEC

\section{Radiation/ Whole Breast Radiation}

Target definition includes majority of the breast tissue. A uniform dose distribution and minimal normal tissue toxicity are the goals. The breast should receive a dose of 45 - 50 Gy in 1.8 - 2 Gy per fraction or 42.5 Gy at 2.66 Gy per fraction. A boost to the tumour bed is recommended in patients at higher risk (age $<50$ and high-grade disease).

\section{Chest Wall Radiation (Including Breast Reconstruction)}

The target includes the ipsilateral chest wall, mastectomy scar and drain sites where possible.

\section{Regional Nodal Radiation}

For the paraclavicular and axillary nodes, prescription depth varies based on the size of the patient. For internal mammary node identification, the internal mammary artery and vein location can be used as a surrogate for the nodal locations. Dose is 50 - $50.4 \mathrm{~Gy}$, given as 1.8 - 2.0 Gy fraction size $( \pm$ scar boost at 2 Gy per fraction to a total dose of approximately 60 Gy); given for 5 days per week. If internal mammary lymph nodes are clinically or pathologically positive, radiation therapy should be given. Otherwise, treatment to these nodes is the discretion of the oncologist.

\section{Breast Reconstruction}

Surgical Options for Breast Reconstruction following Mastectomy include-

- Procedures that incorporate breast implants (i.e. Tissue expander placement followed by implant placement, immediate implant placement).

- Procedures that incorporate autologous tissue transplantation (i.e. Pedicled TRAM flap, fat grafting, various microsurgical flaps from the abdomen, back, buttocks and thigh).

- Procedures that incorporate both breast implants and autologous tissue transplantation (e.g. Latissimus Dorsi Flaps).

Breast reconstruction following mastectomy can commence at the same time as mastectomy ("immediate") or at some time following the completion of cancer treatment ("delayed"). In many cases, breast reconstruction involves a staged approach as:

- Surgery on the contralateral breast to improve symmetry.

- $\quad$ Revision surgery involving the breast and/or donor site.

- Nipple and areola reconstruction and tattoo pigmentation. 


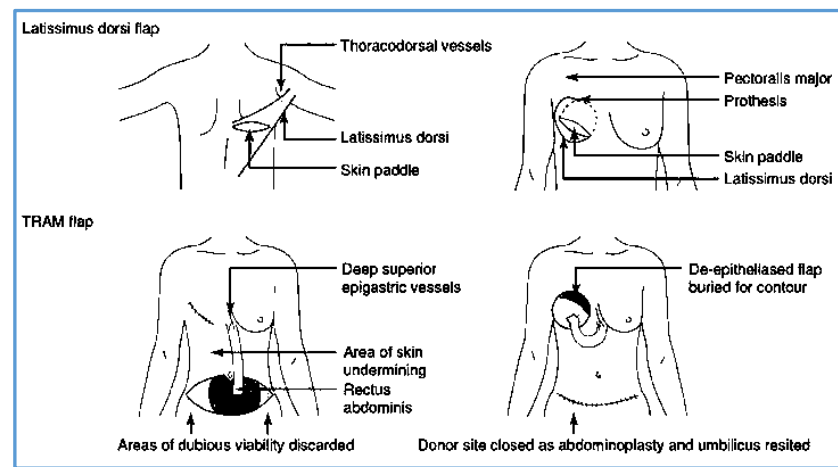

Diagrammatic Representation of Breast Reconstruction (LD Flap and TRAM Flap)

\section{REFERENCES}

[1] Podoloff DA, Advani RH, Allred C, et al. NCCN task force report: positron emission tomography (PET)/computed tomography (CT) scanning in cancer. J Natl Compr Canc Netw 2007;5 Suppl 1:S1-S22.

[2] Rosen EL, Eubank WB, Mankoff DA. FDG PET, PET/CT, and breast cancer imaging. Radiographics 2007;27 Suppl 1:S215-29.

[3] Van der Hoeven JJM, Krak NC, Hoekstra OS, et al. 18F2-fluoro-2-deoxy-d-glucose positron emission tomography in staging of locally advanced breast cancer. Journal of Clinical Oncology 2004;22(7):12539.

[4] Cianfrocca M, Goldstein LJ. Prognostic and predictive factors in early-stage breast cancer. Oncologist 2004;9(6):606-16.

[5] Madarnas $Y$, Trudeau M, Franek JA, et al. Adjuvant/neoadjuvant trastuzumab therapy in women with HER-2/neu-overexpressing breast cancer: a systematic review. Cancer Treat Rev 2008;34(6):53957.

[6] Allred DC, Carlson RW, Berry DA, et al. NCCN Task Force Report: estrogen receptor and progesterone receptor testing in breast cancer by immunohistochemistry. J Natl Compr Canc Netw 2009;7 Suppl 6:S1- S21, quiz S22-3.

[7] Hammond ME, Hayes DF, Dowsett M, et al. American Society of Clinical Oncology/College of American Pathologists guideline recommendations for immunohistochemical testing of estrogen and progesterone receptors in breast cancer. J Clin Oncol 2010;28(16):2784-95.

[8] Harris L, Fritsche H, Mennell R, et al. American Society of Clinical Oncology 2007 update of recommendations for the use of tumor markers in breast cancer. J Clin Oncol 2007;25(33):5287-312.

[9] Valero VV, Buzdar AU, Hortobagyi GN. Locally advanced breast cancer. Oncologist 1996;1(1 \& 2):817.

[10] Jeffrey SS, Lonning PE, Hillner BE. Genomics-based prognosis and therapeutic prediction in breast cancer. J Natl Compr Canc Netw 2005;3(3):291-300.

[11] Rathore AS, Kumar S, Konwar R, et al. Presence of CD3+ tumour infiltrating lymphocytes is significantly associated with good prognosis in infiltrating ductal carcinoma of breast. Indian J Cancer 2013;50(3):23944.
[12] Mieog JS, van der Hage JA, van de Velde CJ. Neoadjuvant chemotherapy for operable breast cancer. Br J Surg 2007;94(10):1189-200.

[13] Lee MC, Newman LA. Management of patients with locally advanced breast cancer. Surg Clin North Am 2007;87(2):379-98.

[14] Kuehn T, Vogl FD, Helms G, et al. Sentinel-node biopsy for axillary staging in breast cancer: results from a large prospective German multi-institutional trial. Eur J Surg Oncol 2004;30(3):252-9.

[15] Fisher B, Bryant J, Wolmark N, et al. Effect of preoperative chemotherapy on the outcome of women with operable breast cancer. J Clin Oncol 1998;16(8):2672-85.

[16] Hortobagyi GN, Singletary SE, Strom EA. Locally advanced breast cancer. In: Harris JR, Lippman ME, Morrow M, et al. eds. Diseases of the breast. Philadelphia: Lippincott Williams \& Wilkins 2004.

[17] Ghezzi P, Magnani S, Rinaldini $M$, et al. Impact of follow-up testing on survival and health-related quality of life in breast cancer patients. A multicenter randomized controlled trial. The GIVIO Investigators. JAMA 1994;271(20):1587-92.

[18] Del Turco RM, Palli D, Cariddi A, et al. Intensive diagnostic follow-up after treatment of primary breast cancer: a randomized trial. National Research Council Project on Breast Cancer follow-up. JAMA 1994;271(20):1593-7.

[19] Smith TJ, Davidson NE, Schapira DV, et al. American Society of Clinical Oncology 1998 update of recommended breast cancer surveillance guidelines. J Clin Oncol 1999;17(3):1080-2.

[20] Zell JA, Tsang WY, Taylor TH, et al. Prognostic impact of human epidermal growth factor-like receptor 2 and hormone receptor status in inflammatory breast cancer (IBC): analysis of 2, 014 IBC patient cases from the California Cancer Registry. Breast Cancer Res 2009;11(1):R9.

[21] Gianni L, Eiermann W, Semiglazov V, et al. Neoadjuvant chemotherapy with trastuzumab followed by adjuvant Trastuzumab versus neoadjuvant chemotherapy alone, in patients with HER2-positive locally advanced breast cancer (the NOAH trial): a randomised controlled superiority trial with a parallel HER2-negative cohort. The Lancet 2010;375(9712):377-84.

[22] Motwani SB, Strom EA, Schechter NR, et al. The impact of immediate breast reconstruction on the technical delivery of postmastectomy radiotherapy. Int J Radiat Oncol Biol Phys 2006;66(1):76-82.

[23] Schneeweiss A, Chia S, Hickish T, et al. Pertuzumab plus trastuzumab in combination with standard neoadjuvant anthracycline-containing and anthracycline-free chemotherapy regimens in patients with HER2-positive early breast cancer: a randomized phase II cardiac safety study (TRYPHAENA). Ann Oncol 2013;24(9):2278-84.

[24] Tolaney SM, Barry WT, Dang CT, et al. A phase II study of paclitaxel (T) and trastuzumab (H) (APT trial) for node-negative, HER2-positive breast cancer (BC). San Antonio Breast Cancer Symposium. Cancer Res 2013;73(Suppl 24):Abstract nr S1-04. 
[25] Gianni L, Pienkowski T, Im YH, et al. Efficacy and safety of neoadjuvant pertuzumab and trastuzumab in women with locally advanced, inflammatory, or early HER2- positive breast cancer (NeoSphere): a randomised multicentre, open-label, phase 2 trial. Lancet Oncol 2012;13(1):25-32.
[26] Toth BA, Forley BG, Calabria R. Retrospective study of the skin-sparing mastectomy in breast reconstruction. Plast Reconstr Surg 1999;104(1):77-84. 